\title{
Modeling for Vibrio fischeri quorum sensing phenomenon based on Multicompartmental Gillespie algorithm
}

\author{
Ji Hu${ }^{1, a}$, Xiaoping Wang ${ }^{1, b}$, Xiaoping Xue ${ }^{1, c}$ \\ ${ }^{1}$ School of Electronics and Information Engineering, Tongji University, Shanghai, China \\ aweilai10000@126.com, bxpwang01@163.com, ‘xuexp@tongji.edu.cn
}

Keywords:Biological systems; Quorum Sensing; Multicompartmental Gillespie algorithm; Formal Modeling and Analysis

\begin{abstract}
Our research purpose is abstracting computational model from the structure and function as well as collaboration among the biolo58gical cells. Quorum sensing P system means bacteria produce specific signaling molecules spontaneously and sense the change in their concentration. In this paper, we use multicompartmental Gillespie algorithm to model and simulate Vibrio fischeri's quorum sensing phenomenon. We put forward an algorithm considering communication and synchronization of each reaction space in a multi-environment $p$ system, whose effectiveness is verified by simulation tools such as CellDesigner and Scilab. At last we propose an idea of using this P System into Multi-Agent System.
\end{abstract}

\section{Introduction}

Membrane computing[1], as a new branch of natural computing, is a computational model at the cellular level to simulate natural processes based on the life process of membrane, whose biggest advantage is the availability of parallel computation. It is distributed, parallel, non-deterministic and easy to implement. In fact, flows among the membrane materials can be viewed as sending message in the computer system, and Quorum sensing is a specific form of mutual communication among bacterial cells.

Membrane computing research is started in 1998, when Gheorghe Paun, a Romanian scientist and academician of European Academy of Science, abstracted the first membrane computation model according to the cells' reaction mechanism in face of different chemical substances. Membrane computation attracts a lot of concern since its introduction, and researchers gradually put forward different types of P System, which can roughly be divided into three categories: Cell-like P System, Tissue-like P System and Neural-like P System. Cell-like P System simulates the structure and function of cells, including eukaryotic cells; Tissue-like P System, as an important expansion of P model, places multiple cells in the same environment, communication among cells or between cells and environment is conducted through transfer rules; Neutral-like P System, the latest proposed computational model and a heated topic of membrane computation research, borrowed the idea from biological nerve system and uses neuronal cells.

$\mathrm{P}$ system in modeling process is defined as a model with hierarchical structure, in which different levels represent different environment and reaction space in cells. The Schematic of single-cell $\mathrm{P}$ System is shown in Fig. 1, where the outermost membrane is called the skin membrane, which separates the system from external environment.

Quorum sensing is the group behavior that bacteria spontaneously generate, release specific signaling molecules, perceive changes in concentrations and adjust their behavior. Bacteriological quorum sensing is involved in the regulation of biological function including the pathogenicity capacity of human, fauna and flora. In such a multi-environment model, quorum sensing is driven by the signal bacteria in the environment, and the signaling molecules are limited to a specific environment. P system modeling using the mechanism based on quorum sensing is a new method of $\mathrm{P}$ modeling. It works well with the maximized-parallelism and uncertainty of real computing system. 
In this paper, we first simulate the quorum sensing in Vibrio fischeri using Cell Designer as the modeling tool. Secondly, We establish population P System based on Vibrio Fischeri, which defines a complete set of reaction rules and multiple sets, and utilize the mechanism of quorum sensing itself. We apply multicompartmental Gillespie algorithm to Scilab simulation tool, in order to simulate the parallelism and uncertainty in the intracellular reactionn in population P System. Finally, we combine population P System and Multi-Agent System to explore the areas that this system can be applied to.

\section{Related Works}

Quorum sensing is a special form of communication among bacteria cells, which allows an entire population of bacteria cells communicate in order to regulate the expression of certain or specific genes in a coordinated way according to the size of the population. Bacteria pass messages to each other through signaling molecules, so that it can detect the density of population. As the number of bacteria reaches a certain level, it will launch a series of corresponding gene expression, so as to control and adjust the bacterial community [2]. Quorum sensing phenomenon is first discovered in Gram negative bacteria, which is also the simplest, followed by a more complex one, Vibrio fischeri. P System, based on quorum sensing [3], inspired by quorum sensing mechanism, is a new type of population $\mathrm{P}$ system. In the perspective of biology, a population $\mathrm{P}$ system model is an abstraction of the way population aggregate, which is more complex than its component parts. The population $\mathrm{P}$ system is composed of many individual components that cooperate and interact with each other as well as with the environment. The communication medium, such as small molecules, ions and molecules, usually slightly more than one, is often disordered as well. In P system, we use small letters to represent these substances, which can be expressed by a multi-set in cell membrane. A multi-set means the same elements can exist in a collection.

In this paper, we present a model of the Quorum Sensing System in Vibrio fischeri using quorum-sensing-based population P System. In this class of P system, we should take not only single cell's reaction but also the communication among different cells in the whole environment into consideration. We define this population $\mathrm{P}$ system as a graph $\mathrm{G}=(\mathrm{V}, \mathrm{S})$ whose nodes $\mathrm{V}$ represent environments. These environments are connected by the edges $\mathrm{S}$ which define the links between them. The Schematic of population P System is shown in Fig. 2.
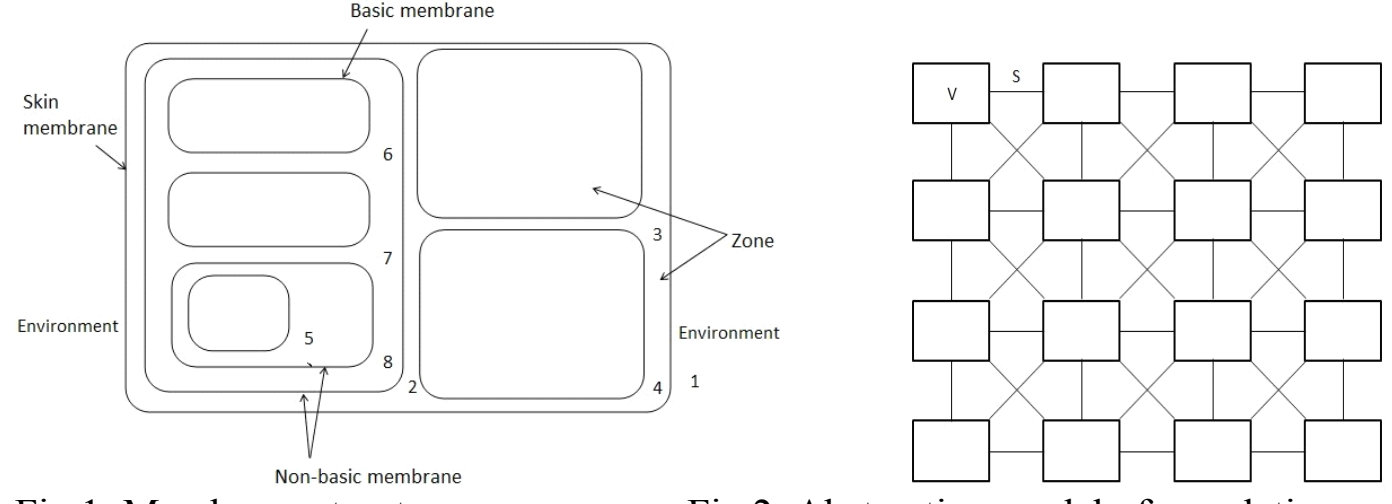

Fig.1. Membrane structure

Fig.2. Abstraction model of population $\mathrm{p}$ system

In the process of simulation, we use the simulation tools based on Multicompartmental Gillespie Algorithm, which is an improvement of Stochastic Simulation Algorithm [5] (SSA). The basic assumption of stochastic simulation algorithms is that the reaction system is an equal concentration system, while a multienvironment reaction system cannot meet this requirement, so the critical issue of multicompartmental simulation algorithm is to solve the problem of how to determine the steps of reaction process and how to coordinate and synchronize the simulation process between several environments.

- Steps of multicompartmental reaction system:

There is a uniform time in the system using this algorithm, which means each reaction chamber has a uniform timeline. Multicompartmental Gillespie Algorithm simulates the parallel multienvironment 
reaction using a serial method. Firstly we calculate $\tau$ of each compartmentalization using SSA, and then we can get all parameters of the reaction system $\left(\tau_{\mathrm{m}}, \mathrm{j}_{\mathrm{m}}, \mathrm{M}\right) \mathrm{M} \in 1 \ldots \mathrm{k}$ :

M represents the number of compartmentalization with a total of $k$

$\checkmark \quad \mathrm{j}_{\mathrm{m}}$ means the next to be triggered reaction channel in compartmentalization $\mathrm{M}$. $\mathrm{j}_{\mathrm{m}}$ is also calculated by SSA.

$\checkmark \quad \tau_{\mathrm{m}}$ is calculated by SSA to work out the time interval before the next reaction.

After calculating we can sort the parameter tuples by $\tau_{\mathrm{m}}$ and find out the minimal $\tau_{\mathrm{m}}$. The minimal $\tau_{\mathrm{m}}$ indicates the reaction happens earliest so that the issue of reaction step is solved.

- Coordination and synchronization of reaction system:

Since each reaction in own compartmentalization may influence concentrations and trigger conditions of other compartmentalization, we reset the timeline in the serial simulation process after one reaction finished, and then recalculate the concentrations and changes of reactants both in own and other compartmentalization. The coordination and synchronization problem will be solved through this approach.

\section{Modeling and Verification}

Theory Foundation. The quorum sensing phenomenon was first investigated in the bacterium Vibrio fischeri. Vibrio fischeri exists in free floating way in ecological systems, and can glow with a particular form of aquatic organisms. When Vibrio fischeri is symbiotic with specific light-emitting aquatic organisms, it will shine in the water. Quorum sensing mechanism in Vibrio fischeri relies on the biosynthesis of specific protein signaling molecule 3-OXO-C6-HSL (we will refer to it as OHHL). When the number of bacteria in the whole colony is at a relatively low level, bacteria will generate little signaling molecule and diffuse little to the surroundings environment. In contract, when the number is at a relatively high level, the bacteria can produce a large number of signaling molecules OHHL and pass them to the surroundings; at the same time the signal accumulates in the environment will diffuse into bacterial cells. Bacterial sensing system senses to reach the threshold of signaling molecules, which means the population is large enough, then it will trigger a series of its own gene transcription. The signaling molecules can interact with the LuxR protein to form the complex LuxR-OHHL and then this complex will combine with LuxBox in DNA segment. These two reactions will cause the transcription of luminescence genes called luxCDABE. In this way OHHL and LuxR are said to be auto-inducer because they trigger their own synthesis.

System Modeling. Firstly we use the population P System to model the quorum sensing phenomenon of Vibrio fischeri, we define the tuple as follows:

$\mathrm{ME}(\mathrm{N})=\left(\{\mathrm{OHHL}\}, \mathrm{G},\{\varnothing, \mathrm{e}\}, \ldots\{\varnothing, \mathrm{e}\}, \mathrm{R}_{\mathrm{e}}, \ldots, \mathrm{R}_{\mathrm{e}}, \mathrm{N}, \Sigma\right)$

- Alphabet $\{\mathrm{OHHL}\}$ represents the signaling molecules,

- $\mathrm{G}$ represents the population structure which consists of 16 environment-s depicted in figure 3,

- $\{\varnothing, \mathrm{e}\}$ indicates the initial state of environment which is marked as e,

- Re represents the reaction rules in environment,

- $\quad \mathrm{N}$ represents the number of bacteria in the whole population

- $\Sigma$ represents the formal definition of Vibrio fischeri in the reaction environment and has a total of $\mathrm{N}$ copies. $\Sigma$ is defined as follows:

$\Sigma=\left(\mathrm{O}, \mu, \mathrm{M}_{1}, \mathrm{M}_{2}, \ldots, \mathrm{M}_{\mathrm{n}}, \mathrm{R}_{1}, \ldots, \mathrm{R}_{\mathrm{n}}\right)$

- O represents a non-empty finite alphabet, each symbol of which represents a substance in membrane. Vibrio fischeri quorum sensing mechanism includes LuxR-OHHL, LuxBox, LuxR and 
OHHL other objects.

$\checkmark \quad \mu$ is a membrane structure containing $n$ membranes, $H=\{1,2, \ldots, n\}$ is the label set of $\Sigma$.

$\mathrm{M}_{\mathrm{i}}(1 \leq \mathrm{i} \leq \mathrm{n})$ represents the collection of objects in membrane $\mathrm{I}$, which is expressed by a multi-set. $\mathrm{M}_{\mathrm{i}}=\lambda$ means there is no objects.

$\mathrm{R}_{\mathrm{i}}(1 \leq \mathrm{i} \leq \mathrm{n})$ indicates the evolution rules of membrane $\mathrm{i}$. The rule form is $\mathrm{u} \rightarrow \mathrm{v}$ or $\mathrm{u} \rightarrow \mathrm{v} \delta$, and $\mathrm{u} \in \mathrm{O}^{+}, \mathrm{v} \in(\mathrm{O} \times \mathrm{Tar}), \mathrm{Tar}=\{$ out, h-ere, in $\}$.

Secondly, we define the complete $\mathrm{P}$ System reaction rule depending on the Vibrio fischeri quorum sensing mechanism. Reaction rule consists of Re (reaction rules in the external environment) and $\mathrm{Ri}$ (reaction rules inside bacteria):

- In this simulation, bacteria is the identical copies of each other, so they have the same inner rules. $\mathrm{R}_{\mathrm{i}}$ is defined as follows:

Rule1: [LuxBox] $\rightarrow$ [LuxBox, OHHL]

Rule2: [LuxBox] $\rightarrow$ [LuxBox, LuxR]

$\checkmark \quad$ Rules 1 and 2 indicates that when the number of bacteria is at a lower level, bacteria will generate LuxBox spontaneous and slowly.

Rule3: [LuxR, OHHL] $\rightarrow$ [LuxR-OHHL]

Rule4: [LuxR-OHHL] $\rightarrow$ [LuxR, OHHL]

- Rule 3 and 4 show that signaling molecules can interact with the LuxR protein to form the complex LuxR-OHHL, and LuxR-OHHL will decompose into LuxR and OHHL in return.

Rule5: [LuxBox , LuxR-OHHL] $\rightarrow$ [ LuxBox-LuxR-OHHL ]

Rule6: [LuxBox-LuxR-OHHL] $\rightarrow$ [ LuxBox , LuxR-OHHL ]

Rules 5 and 6 indicate that the complex LuxR-OHHL acts as a transcription factor binding to the regulatory region of DNA called LuxBox. The complex LuxR-OHHL can also dissociate from the LuxBox.

Rule7: [LuxBox-LuxR-OHHL] $\rightarrow$ [LuxBox-LuxR-OHHL, OHHL]

Rule8: [LuxBox-LuxR-OHHL] $\rightarrow$ [LuxBox-LuxR-OHHL, LuxR]

$\checkmark \quad$ Rules 7 and 8 indicate the fact when there is LuxBox-LuxR-OHHL in the population, it will generate a large number of signaling molecules OHHL, LuxR and itself.

Rule9: [OHHL] $\rightarrow$ OHHL [ ]

$\checkmark \quad$ Rule 9 indicates that the signal molecules diffuse into the environment from the cell membrane.

Rule10: [OHHL] $\rightarrow[$ ]

Rule11: [LuxR] $\rightarrow$ [ ]

Rule12: [LuxR-OHHL] $\rightarrow[$ ]

- Rules 10, 11, 12 indicate that the signaling molecule OHHL, LuxR proteins and transcriptional gene LuxR-OHHL will be self-decomposed at a certain rate.

- Reaction rules in the external environment $R_{\mathrm{e}}$ is defined as follows:

Rule13: OHHL [ ] $\rightarrow$ [ OHHL ]

environment.

Rule14: [OHHL] e $\rightarrow$ [ ] e

Rule 14 represents the signaling molecules decompose in the environment e.

Rule15: [OHHL] $\mathrm{e}_{1}-$ [ ] e1 $\rightarrow$ [ ] $\mathrm{e}_{2}-$ [OHHL] e2

$\checkmark \quad$ Rule 15 indicates that the signaling molecules move from environment e1 to environment e1 e2.

Simulation and Verification. According to the formal definition and rules described above, we simulate the quorum sensing of Vibrio fischeri and find out different quorum sensing phenomena depending on whether the number of bacteria population reaches a threshold. In this way we specify the quorum sensing mechanisms from simulation results and prove Multicompartmental Gillespie algorithm [4] is effective.

Firstly we implement rules and definition in CellDesigner. CellDesigner is a structured diagram editor for drawing gene-regulatory and biochemical networks. Networks are drawn based on the 
process diagram, with graphical notation system proposed by Kitano, and are stored using the Systems Biology Markup Language (SBML), a standard for representing models of biochemical and gene-regulatory networks. Toolbar and user interface of CellDesigner are shown in Fig. 3.

Secondly, input the modeling results of the previous step into simulation tool- Scilab, which has already integrated Multicompartmental Gillespie algorithm. The simulation result when bacterium has 250 copies is shown in figure 5, where green line represents the number of bacteria and blue line represents the number of signaling molecules OHHL, the horizontal axis is the timeline. It can be seen in Fig. 5 that at the beginning of the simulation, the population is still in the process of communication, the bacteria can't detect the size of population, so the number of both bacteria and signaling molecules is increasing. After a period of time, bacteria judge the number of activated population to be greater than the threshold by detecting the number of signaling molecules OHHL. Therefore, in a short time the whole population reflects the quorum sensing phenomenon. The number of activated bacteria and signaling molecules is going to be stable so that the population of bacteria finishes self-regulation.

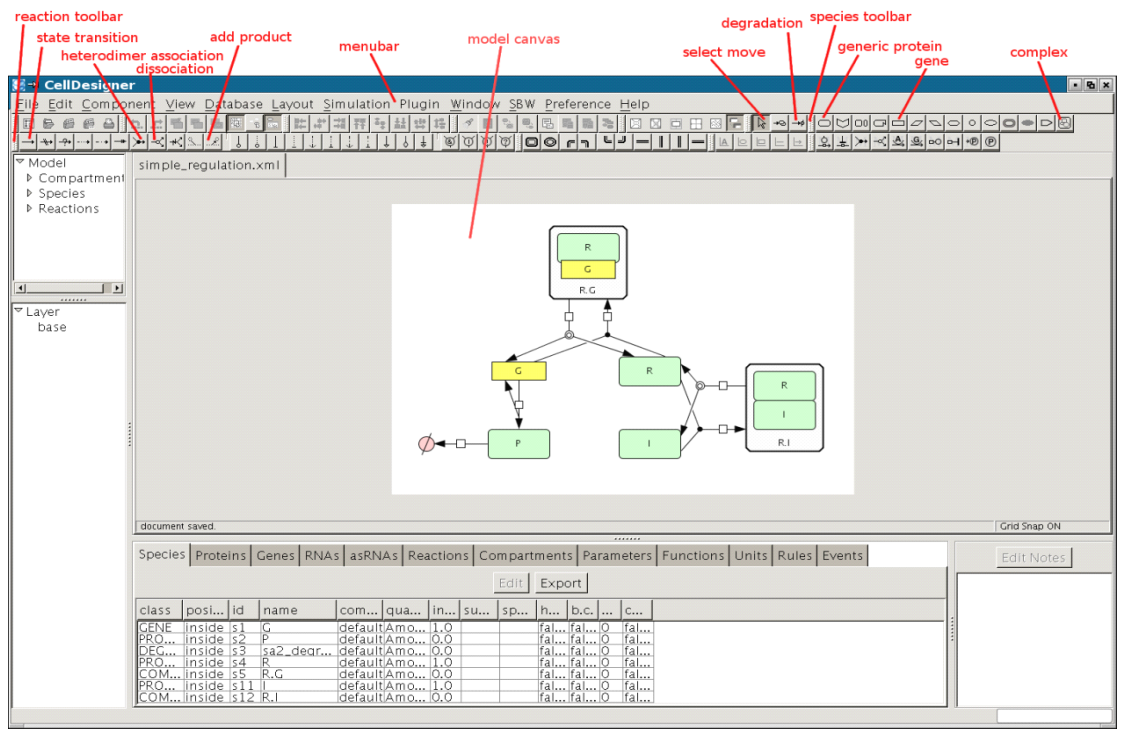

Fig. 3 CellDesigner user interface

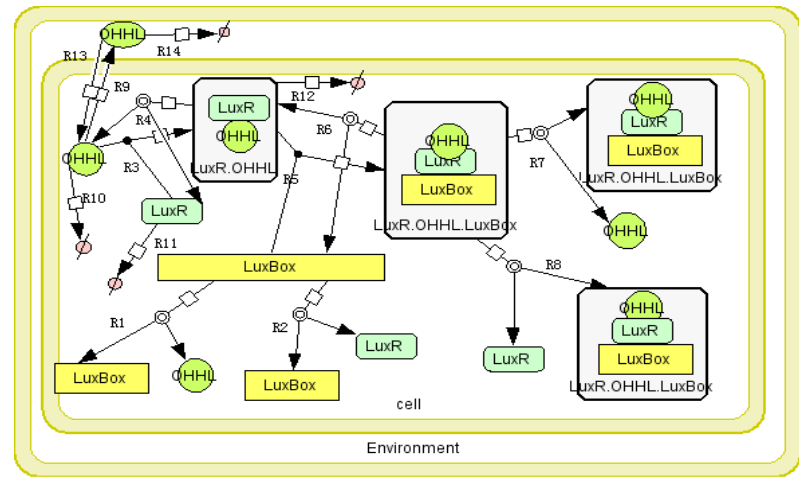

Fig. 4 Graphic model of quorum sensing

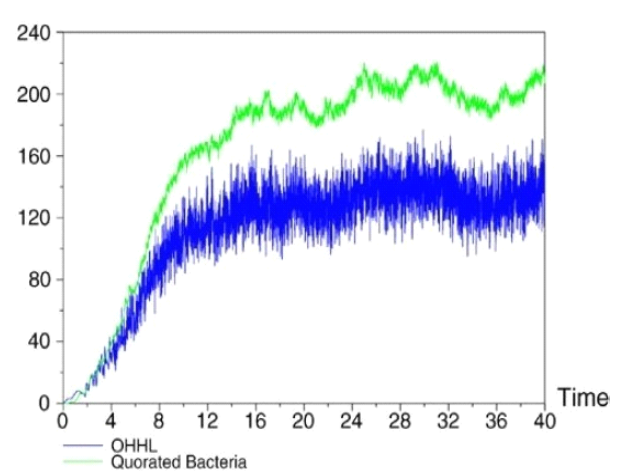

Fig. 5 Number changing of OHHL and bacteria

As we can see from the simulation result, colony of bacteria is sensitive to whether the number of colonies reach threshold by communication with each other and the communication rule is based on Multicompartmental Gillespie algorithm. When the number of bacteria population is increasing, bacteria will regulate themselves through quorum sensing mechanism. As the result of simulation is corresponding to the actual phenomenon, Multicompartmental Gillespie algorithm is proved to be effective.

\section{Application Prospects}

At the present stage when researches related to the application of quorum sensing is still rare, this paper puts forward the idea of abstract modeling using combining Tissue-like P System and 
Multi-Agent System. Multi-Agent System is a loosely coupled network of agents, which solves problems through interaction and collaboration. Each of these agents is autonomous, and can be developed by different designing methods and languages. In particular, thinking agent views each agent as an intelligent awareness system, that is, it achieves multi-agent collaboration through the communication between the agents and internal self-change. The basic structure of thinking agent is shown in Fig. 6.

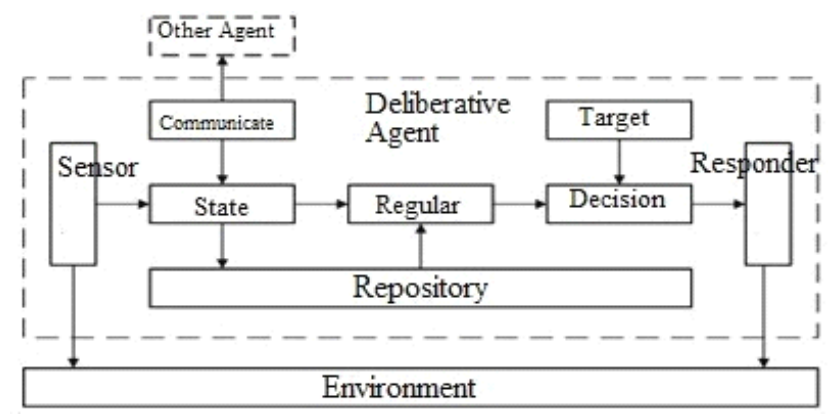

Fig. 6 Model of Deliberative Agent

The collaboration among thinking agents is quite similar to the quorum sensing mechanism. The limited information that the collaboration in Multi-Agent System relies on can be viewed as signaling molecules in colonies; single agent is viewed as a single membrane in Tissue-like P System; the internal state of agent can be converted into a limited character set in cell membrane; and the knowledge base in agent model is equivalent to the reaction rule in membrane. Therefore, we work out the formalized modeling of Multi-Agent System using Tissue-like P System, and call the proxy nodes in Multi-Agent System randomly using Multi-Room Randomized Algorithm in order to realize coordination and communication among agents.

\section{Conclusion}

As the development of traditional computer science facing bottleneck day by day, searching for alternative to replace traditional model and algorithm is more and more urgent. Computer science using bionic principle is quite favored by researchers for its high parallelism and low energy consumption. In this paper, we put forward a modeling method to simulate the quorum sensing mechanism in Tissue-like P System, and verified the validity of Multi-compartmental Gillespie, an algorithm of Multi-Agent System, together with simulation tools. Although the detail of quorum sensing is a complex mechanism, the basic principle behind is simple. The model we worked out helps further understand the complexity and functions of quorum sensing in order to make its favorable characteristics better applied in reality.

\section{References}

[1] PÁun Gh, Membrane Computing, An Introduction. Berlin: Springer, 2002.

[2] Miller M B, Bassler B L. Quorum sensing in bacteria[J]. Annual Reviews in Microbiology, 2001, Vol. 55, No. 1, pp. 165-199..

[3] Bernardini, F, Gheorghe, M. (2004) Population P systems. J. Universal Computer Sci., 10, pp. 509-539.

[4] Bernardini, F. Membrane, Systems for Molecular Computing and Biological Modelling: [PhD Dissertation]. Sheffield, UK: The Univ. of Sheffield, 2005.

[5] Gillespie D.T, A general method for numerically simulating the stochastic time evolution of coupled chemical reactions . Journal of computational physics, Vol. 22, No. 4, 1976, pp. 403-434.

[6] Stone P, Veloso M,Multiagent systems: A survey from a machine learning perspective, Autonomous Robots, Vol. 8, No. 3, 2000, pp. 345-383. 